\title{
DISTANCE MEASUREMENT ERRORS IN SILENT FM-CW SONAR WITH MATCHED FILTERING
}

\section{Jacek Marszal, Roman Salamon}

Gdansk University of Technology, Faculty of Electronics, Telecommunication and Informatics, Department of Maritime Electronic Systems, G. Narutowicza 11/12, 80-233 Gdansk, Poland (هjacek.marszal@eti.pg.gda.pl, +48 5834723 36)

\begin{abstract}
The secretiveness of sonar operation can be achieved by using continuous frequency-modulated sounding signals with reduced power and significantly prolonged repeat time. The application of matched filtration in the sonar receiver provides optimal conditions for detection against the background of white noise and reverberation, and a very good resolution of distance measurements of motionless targets. The article shows that target movement causes large range measurement errors when linear and hyperbolic frequency modulations are used. The formulas for the calculation of these errors are given. It is shown that for signals with linear frequency modulation the range resolution and detection conditions deteriorate. The use of hyperbolic frequency modulation largely eliminates these adverse effects.
\end{abstract}

Keywords: silent sonar, frequency modulation, continuous wave, matched filtering, distance measurement, errors, Doppler effect.

\section{Introduction}

When used in military applications, sonars and radars should be difficult to intercept. Stealth is a feature offered by silent radars using continuous signals and linear frequency modulation [1-6]. Because they operate at significantly reduced power, compared to pulse radars, the distance between the signal emitted by a silent radar and the enemy's listening system is shorter. Analogous silent sonars are not manufactured. The most likely reason for that is the Doppler effect and the errors it causes in measuring the distance to moving targets [7-9]. This is not an issue with radars, however, because the speed of electromagnetic wave propagation is about 200000 greater than the speed of acoustic wave propagation in water. Despite that, literature on radars covers this aspect extensively, especially the resolution of target distance measurements using pulse sonars and the negative effect of the Doppler effect on detection capacity. These problems are analyzed using the narrowband ambiguity function for selecting signals that will ensure the desired resolution and detection capacity $[6,10]$.

We will analyze errors of silent sonar distance measurements using the broadband ambiguity function [11] because emitted acoustic signals feature a high relative bandwidth. We will look at two types of signals, namely continuous signals with linear and hyperbolic frequency modulation. Frequency modulated continuous wave (FM-CW) radars use the Fourier transform of the product of the signal emitted and echo signal for detection purposes. This article will cover detection with matched filtering. This type of detection is optimal when known signals are received against the background of Gaussian noise [12]. We will use the results of the analysis to determine the parameters of the silent sonar that will ensure that distance measurement errors are acceptable. 


\section{The principle of operation of the silent sonar with matched filtering}

The sonar's transmitter emits a periodical signal $x(t)$ which we will write down as:

$$
x(t)=\sum_{n=0}^{\infty} s(t-n T),
$$

where $s(t) \neq 0$ only in the range $0<t<T$.

We assume that the echo signal $y(t)$ is a delayed and reduced copy of signal $x(t)$, namely:

$$
y(t)=x_{o} x\left[t-\tau_{d}(t)\right],
$$

where $x_{0}$ is the echo signal amplitude, $\tau_{d}(t)$ is a momentary delay of the echo signal versus the transmitted signal equal to:

$$
\tau_{d}(t)=2 \frac{R}{c}-2 \frac{v t}{c} .
$$

In the above formula, $R$ is the distance between the target and the sonar at a moment of time $t=0, c-$ is the speed of propagation of an acoustic wave in water, and $v$ - is the radial of speed at which the target is moving towards the sonar. To simplify further analysis we will assume that over a relatively short time of observation $x_{0}$ is constant.

We substitute relation (3) to formula (1) and obtain:

where

$$
y(t)=x_{o} x\left[d\left(t-\tau_{0}\right)\right],
$$

$$
d=\frac{c+v}{c-v} \cong 1+2 \frac{v}{c}
$$

is a parameter of time compression resulting from the Doppler effect, and $\tau_{0}=2 R / d c$ is the delay of the echo signal. We assume that the delay $\tau_{0}<T$ which helps with interpreting the results of distance to target measurements. Because of time compression the period of function $y(t)$ is $T_{d} \cong T / d$.

If the signal frequency $s(t)$ changes linearly in time, the frequency of signals $x(t)$ and $y(t)$ can be illustrated as shown in Fig. 1 below.

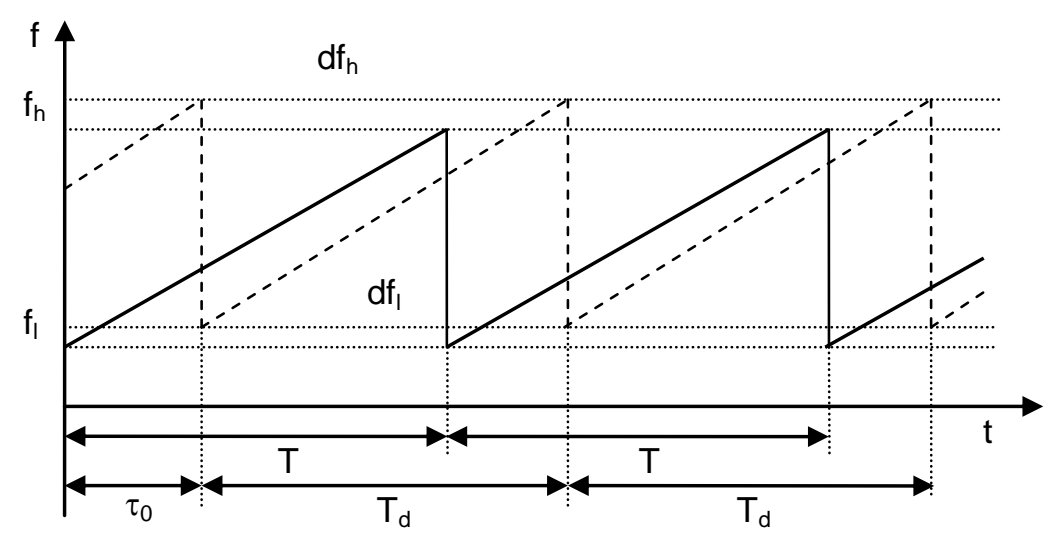

Fig. 1. The frequency of the emitted signal (solid line) and echo signal (dotted line).

The receiver is used for determining the correlation function of the emitted signal and echo signal:

$$
z(t)=\int_{0}^{t} s\left(t^{\prime}\right) y^{*}\left(t^{\prime}-t\right) d t^{\prime}
$$


or for matched filtering in the frequency domain following this formula:

$$
z(t)=\mathfrak{J}^{-1}\left\{S(f) Y^{*}(f)\right\},
$$

where $S(f)$ and $Y(f)$ are Fourier transforms of signals $s(t)$ and $y(t)$. As you know, both are equivalent operations, [12].

Let us next consider two signals with mid frequency $f_{0}$, bandwidth $B$ and duration $T$, equal to the period of function $x(t)$. The signal with linear frequency modulation (LFM) can be written down as:

$$
s(t)=\exp \left[j 2 \pi\left(f_{0}-\frac{B}{2}+\frac{B}{2 T} t\right) t\right]
$$

and the signal with hyperbolic frequency modulation (HFM) as:

$$
s(t)=\exp \left[-j 2 \pi \frac{f_{l} f_{h}}{B} T \ln \left(1-\frac{B}{f_{h} T} t\right)\right],
$$

where $f_{l}=f_{0}-B / 2, f_{h}=f_{0}+B / 2$ are the upper and lower frequency of both signals respectively.

Fig. 2 shows the spectrum of signal $s(t)$ with linear frequency modulation and Fig. 3 shows the same with hyperbolic frequency modulation.

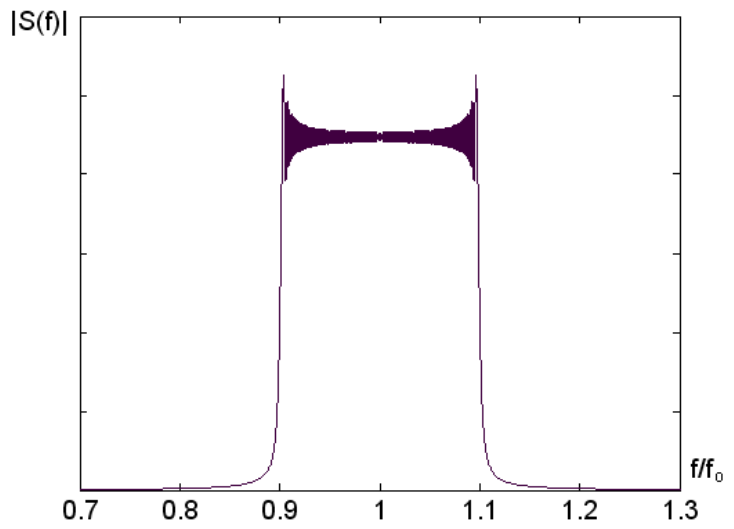

Fig. 2. Module of the LFM signal spectrum.

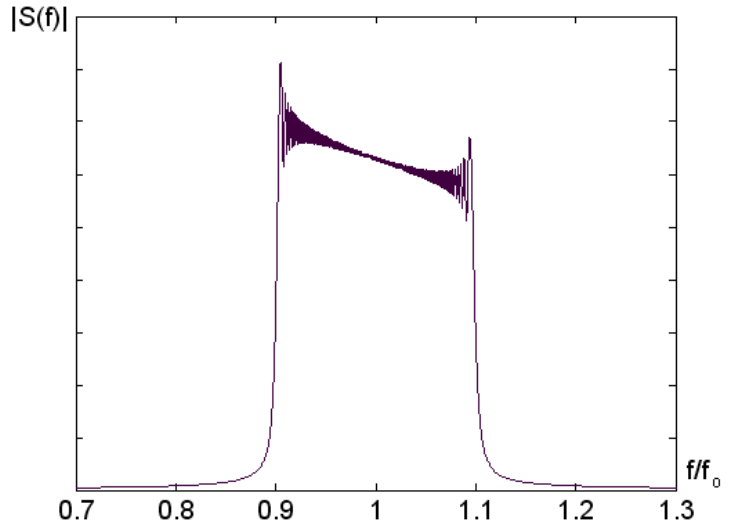

Fig. 3. Module of the HFM signal spectrum.

For $v=0$ at the output of a matched filter the signal we obtain is proportional to the periodically recurring autocorrelation function delayed by $\tau_{0}=2 R / c$. Fig. 4 shows a single period of the LFM signal at the output of a matched filter and Fig. 5 shows a magnified fragment of the signal. Numerical calculations were made for $f_{0}=10 \mathrm{kHz}, B=2 \mathrm{kHz}$ and $T=10 \mathrm{~s}$. The assumed target distance is $R=3 \mathrm{~km}$, and the propagation speed of the acoustic wave where the time scale has been replaced with the distance scale is $c=1500 \mathrm{~m} / \mathrm{s}$.

As you can see from the charts, the maximum of the matched filter output signal occurs exactly at the assumed target distance. The ambiguity of the distance measurement is the result of the correlation function width as shown in Fig. 5 and is inversely proportional to signal spectrum width. For the data used in the charts, the ambiguity defined as the distance between distance $R=3 \mathrm{~km}$ and the contiguous zero of function $z(R)$ is: $\delta R=c / 2 B=37.5 \mathrm{~cm}$. In practice, distances are never measured with this theoretical accuracy (which is usually not necessary at all). This is primarily because we do not know the exact speed of sound in the sea, acoustic wave propagation is not a straight line and noise or other factors do not occur [13]. The speed of sound in the sea varies significantly ranging from about $1450 \mathrm{~m} / \mathrm{s}$ to about $1540 \mathrm{~m} / \mathrm{s}$. As a result, when we use the mean value, the relative error in distance measurement 
can be $\pm 3 \%$. The error can be limited by taking frequent measurements of sound velocity range resolutions in advanced underwater acoustic systems.

$\mathrm{z}(\mathrm{R})$

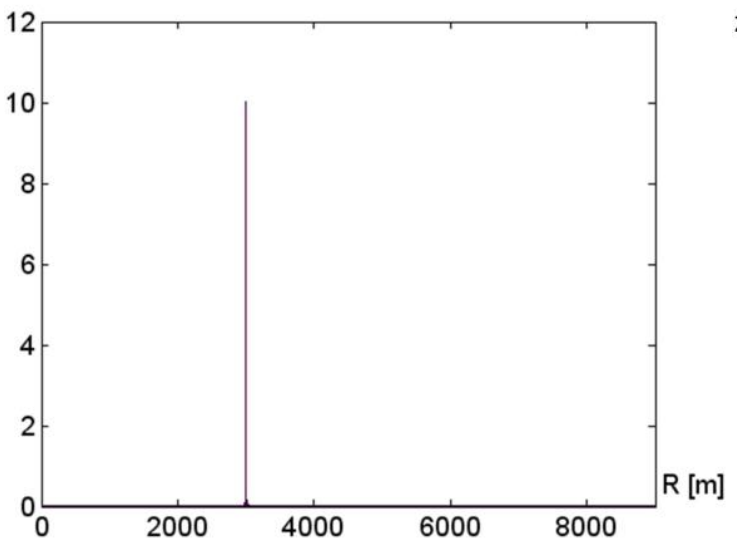

Fig. 4. LFM signal at the matched filter.

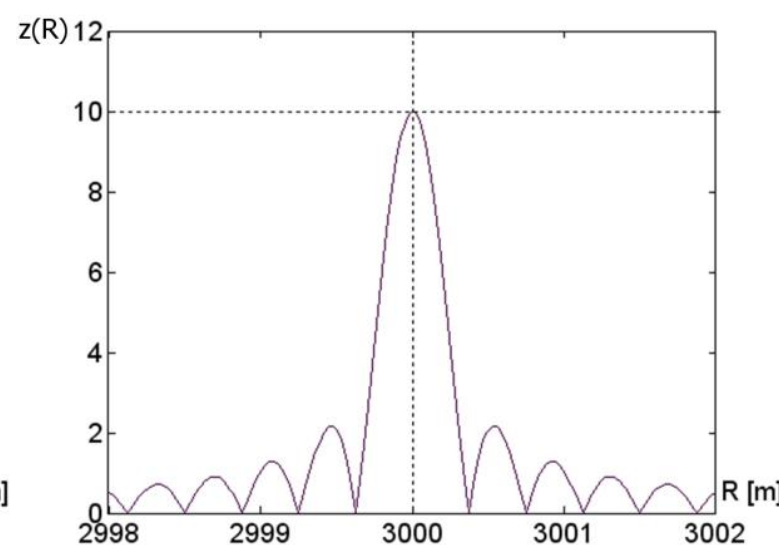

Fig. 5. Magnified fragment of the signal in Fig. 4.

The errors in distance measurements attributed to the above factors are not confined to the silent sonar only and can be seen in all underwater acoustic systems. Further in the paper we will focus on analysing the predominant source of errors in silent sonar distance measurements, i.e. the Doppler effect.

\section{Distance measurement errors for LFM signals}

When analyzing the influence of the Doppler effect on errors in distance measurements, we will use the broadband ambiguity function $A(\tau, d)$ defined as [11]:

$$
A(\tau, d)=\left|\int_{-\infty}^{\infty} s(t) s^{*}[d(t-\tau)) d t\right|
$$

where $\tau$ is the variance between delay $\tau_{0}$ and the moment when the matched filter output signal reaches its maximum. It can be demonstrated that constant delay $\tau_{0}$ has no effect on the results of the analysis [9].

If, for reasons of simplification, we neglect the difference between periods $T$ and $T_{d}$, then by using relation (8), we have:

$$
A(\tau, d)=\left|\int_{-\infty}^{\infty} \exp \left[j 2 \pi\left(f_{0}-\frac{B}{2}+\frac{B}{2 T} t\right) t\right] \exp \left\{-j 2 \pi\left[f_{0}-\frac{B}{2}+\frac{B}{2 T} d(t-\tau)\right] d(t-\tau)\right\} d t\right| .
$$

Following the transformations the above formula reads as follows:

$$
\left.A(\tau, d)=\mid \int_{-\infty}^{\infty} \exp \left\{j 2 \pi(1-d)\left[f_{0}-\frac{B}{2}+\frac{B}{2 T}(1+d) t\right)\right] t\right\} \exp \left(j 2 \pi \frac{B}{T} d^{2} t \tau\right) d t \mid .
$$

Let us note that the expression is the Fourier transform of a signal with linear frequency modulation where the role of frequency $f(\tau, d)$ is taken on by the function:

$$
f(\tau, d)=-d^{2} \frac{B}{T} \tau
$$

As you can see in Fig. 2, the spectrum of the LFM signal is almost rectangular. By differentiating the phase of the signal we obtain: 


$$
\left.f(\tau, d)=(1-d)\left[f_{0}-\frac{B}{2}+\frac{B}{T}(1+d) t\right)\right], \quad \mathrm{t}=[0, \mathrm{~T}] .
$$

The boundary frequencies for time $t=0$ and $t=T / d$ are:

$$
\begin{gathered}
f_{l}(\tau, d)=(1-d)\left(f_{0}-\frac{B}{2}\right), \\
f_{h}(\tau, d)=(1-d)\left(f_{0}+\frac{B}{2}+\frac{B}{d}\right) .
\end{gathered}
$$

By inserting relation (13), and following some simple transformations, we obtain the boundary values of delay $\tau$, with the relevant part of the ambiguity function contained between them. They are respectively:

$$
\begin{gathered}
\tau_{l}=\frac{1-d}{d^{2}} \frac{T}{B}\left(f_{0}-\frac{B}{2}\right) \cong-\frac{2 v}{c} T\left(\frac{f_{0}}{B}-\frac{1}{2}\right), \\
\tau_{h}=\frac{1-d}{d^{2}} \frac{T}{B}\left(f_{0}+\frac{B}{2}+\frac{B}{d}\right) \cong-\frac{2 v}{c} T\left(\frac{f_{0}}{B}+\frac{3}{2}\right) .
\end{gathered}
$$

The mean value of the delay is equal to:

$$
\tau_{m}=\frac{1-d}{d^{2}} \frac{T}{B}\left(f_{0}+\frac{B d}{2}\right) \cong-\frac{2 v}{c} T\left(\frac{f_{0}}{B}+\frac{1}{2}\right)
$$

and the width of the ambiguity function in cross-section $\mathrm{d}=$ const is:

$$
\Delta \tau=\frac{1-d^{2}}{d^{2}} T \cong \frac{4 v}{c} T
$$

For velocity $v=0$ the width of the ambiguity function is not equal to zero because by inserting (12) $d=1$ into the formula we obtain:

$$
A(\tau, 0)=\left|\int_{-\infty}^{\infty} \exp \left(j 2 \pi \frac{B}{T} t \tau\right) d t\right|
$$

By integrating the exponential function within limits $(0, T)$ and following some simple transformation we obtain:

$$
A(\tau, 0)=\left|T \frac{\sin \pi B \tau}{\pi B \tau}\right|
$$

The width of the ambiguity function calculated between the first zeros of the above function is:

$$
\Delta \tau_{\min }=\frac{2}{B} .
$$

This is the lowest width of the ambiguity function which applies to velocities that meet the condition $v<c / 2 B T$. Let us note that an analogous result was obtained in the case of the autocorrelation function shown in Fig. 5.

Fig. 6 shows the cross-sections of ambiguity functions for three values of parameter $d$ and $B / f_{0}=0.2$, which were numerically determined using formula (10). By analogy, just as in the previous analysis, for reasons of simplification the period of both functions was assumed to be $T=1 \mathrm{~s}$. For $d=0.99$ the numerical values of delay and width of the ambiguity function are given based on formulas (19) and (20). 


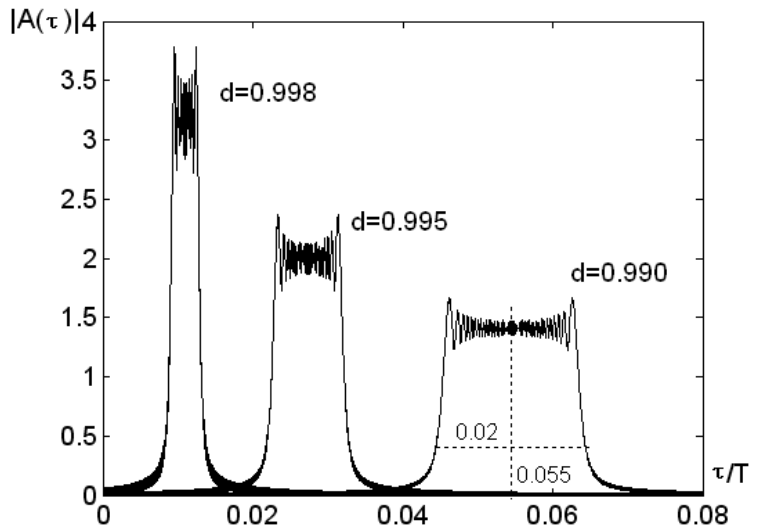

Fig. 6. Cross-sections of the ambiguity function for the LFM signal (simplified version).

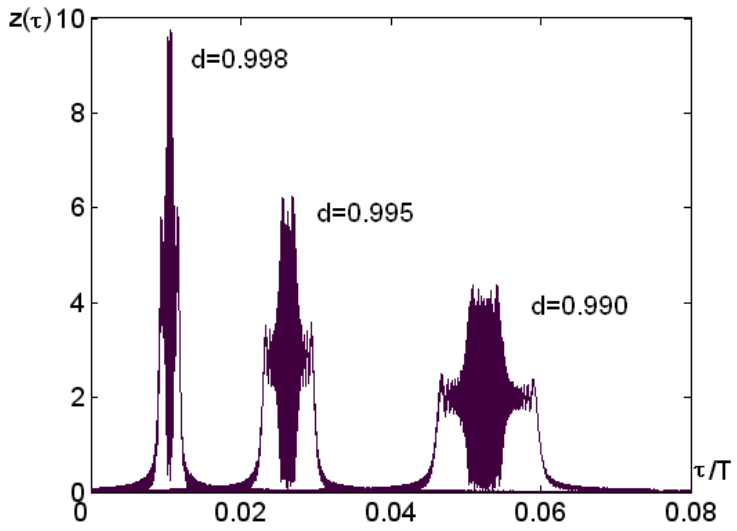

Fig. 7. LFM signals at matched filter output.

As you can see, increases in velocity $v$ have three negative consequences, namely:

- the shift of the ambiguity function increases,

- the function becomes wider,

- the function drops.

Delay $\tau_{m}$ produces errors in distance measurements. The mean error at moment in time $t=T$ when the measurement is taken, based on the formula (19) is equal:

$$
\Delta R_{m}=\frac{c \tau_{m}}{2}-\frac{v T}{2}=-v T \frac{f_{0}}{B} .
$$

When the velocity $v$ sign changes, so does the sign of distance error. If the target is moving closer to the sonar, error $\Delta R_{m}$ has a minus sign. The result is that the distance we have determined is shorter than the actual distance. It is the reverse as the target moves away.

As the ambiguity function widens, the target distance becomes ambiguous. Using formula (20), it can be expressed as:

$$
\delta R=\frac{c \Delta \tau}{2}=2 v T
$$

The formula above also describes the sonar's range resolution, i.e. the minimal difference in the distance between two targets which can be discerned as separate objects.

As the ambiguity function drops, the signal to noise ratio deteriorates which makes target detection less likely.

These effects provide a good basis for selecting the parameters of silent sonar. This will be further discussed in Chapter 5.

The analysis so far is based on the simplified assumption of the sounding signal and echo signal period being identical. As a result, simple formulas could be derived.

Fig. 7 illustrates what happens when we abandon this assumption. It shows matched filter output signals, shifted in time by delay $\tau_{0}=2 R / c$. The velocities of the target and the other parameters of the signals are the same as in Fig. 6. As you can see, the variance between the periods of the sounding signal and echo signal has a significant impact on the signals. The delays of the signals, however, which are important from the perspective of distance measurement errors, are practically unchanged. There is a slight change in signal width but this is the result of signal increase and the energy conservation law.

To recapitulate, the relations derived using the simplified method can be successfully used for sonar design. 


\section{Distance measurement errors for HFM signals}

According to the literature a hyperbolic frequency modulation signal is Doppler effect invariant $[14,15]$. Indeed, the velocity of targets has practically no effect on the signal and in radiolocation a delay in the ambiguity function is almost insignificant. We will demonstrate that distance measurement errors in the case of silent sonar are similar for LFM and HFM signals. To that end we will write down the function $s^{*}[d(t-\tau)]$ from formula (10) using notation (9) of the HFM signal as:

$$
s^{*}[d(t-\tau)]=\exp \left\{j 2 \pi \frac{f_{l} f_{h}}{B} T \ln \left[1-\frac{B}{f_{h} T} d(t-\tau)\right]\right\}, \quad \mathrm{t}=[0, \mathrm{~T}] .
$$

The change in the frequency of the signal in the function of time is:

$$
f(\tau, d)=\frac{-d f_{l}}{1-\frac{B}{f_{h} T} d(t-\tau)} .
$$

So we can easily see that the equivalent notation of the function takes this form:

$$
f(\tau, d)=\frac{-d f_{l}}{1-\frac{B}{f_{h} T}\left(t-\tau-\tau_{d}\right)},
$$

where

$$
\tau_{d}=\frac{1-d}{d} \frac{T f_{h}}{B}
$$

By integrating function (28) and inserting it into formula (26) we obtain:

$$
s^{*}[d(t-\tau)]=\exp \left\{j 2 \pi \frac{f_{l} f_{h}}{B} T \ln \left[1-\frac{B}{f_{h} T} d(t-\tau)\right]+j \varphi\right\},
$$

where $\varphi$ is a certain constant phase.

As a result, the ambiguity function (10) can be written down as:

$$
A(\tau, d)=\left|\int_{-\infty}^{\infty} s(t) s^{*}\left[\left(t-\tau-\tau_{d}\right)\right) d t\right|
$$

It is the auto-correlation function of the HFM signal contained in formula (9) with a shift on the time axis equal $-\tau_{d}$. There is no relation between its maximal value and width and the Doppler effect deviation which in this sense makes it Doppler effect-invariant $[14,15]$. This is illustrated in Fig. 8 which shows the ambiguity function for three values of the parameter $d$ and $B / f_{0}=0.2$. Fig. 9 shows a magnified fragment of the ambiguity function calculated for spectrum width $B=2 \mathrm{kHz}$. As you can see the width of the cross-section is $1 / B$.

The error in target distance measurement is the result of delay $\tau_{d}$. By substituting the value $d=1+2 v / c$ into formula (29) and using the relation (24) we obtain:

$$
\Delta R_{m}=\frac{c \tau_{d}}{2}-\frac{v T}{2}=-v T \frac{f_{0}}{B} .
$$

As you can see, the distance measurement error using hyperbolic frequency modulation is the same as for linear frequency modulation. The Doppler effect does not deteriorate detection conditions because the ambiguity function's maximal values are in no relation to target speed. The sonar's range resolution is also constant and equal to the resolution of a stationary target. 


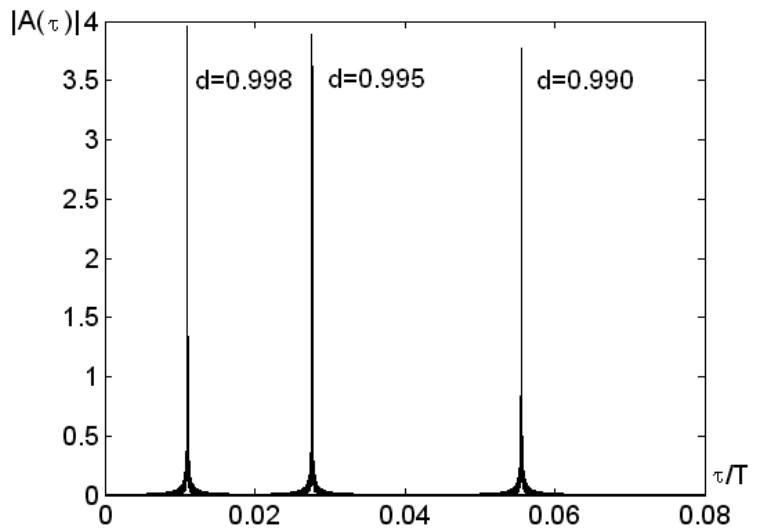

Fig. 8. Cross-sections of the ambiguity function for the HFM signal (simplified version).

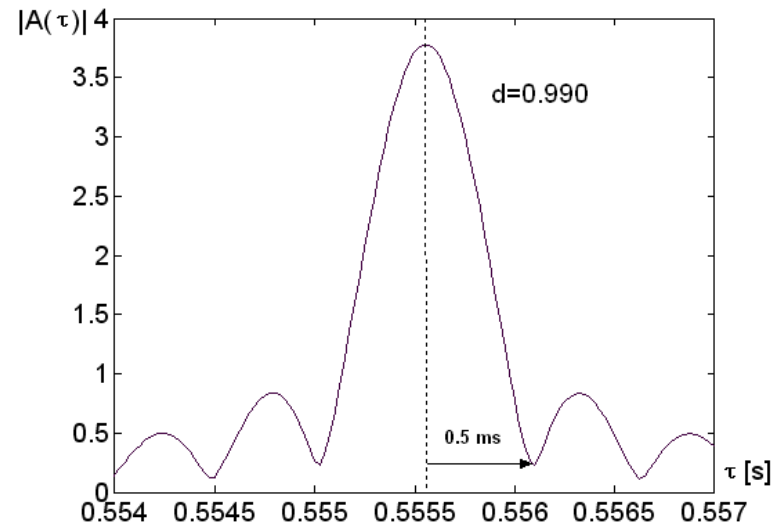

Fig. 9. Magnified fragment of the ambiguity function.

Just as in the case of the LFM signal, the conclusions are derived from a simplified analysis under the assumption that the duration of the HFM signal did not change as the target was moving. Fig. 10 shows HFM signals at the output of a matched filter which take account of the change in the function's duration. A comparison with the signals in Fig. 8 reveals some additional negative consequences of the Doppler effect with the pulses splitting and decreasing. The first consequence deteriorates range resolution while the other affects the conditions of detection. The maximal height of the signals depends on delay $\tau_{0}$ (target distance). It follows the rule that the shorter the delay the bigger the height. In the case of shorter delays side signals diminish which does not, however, affect the distance between them and the tall lines. This is illustrated in Fig. 11.

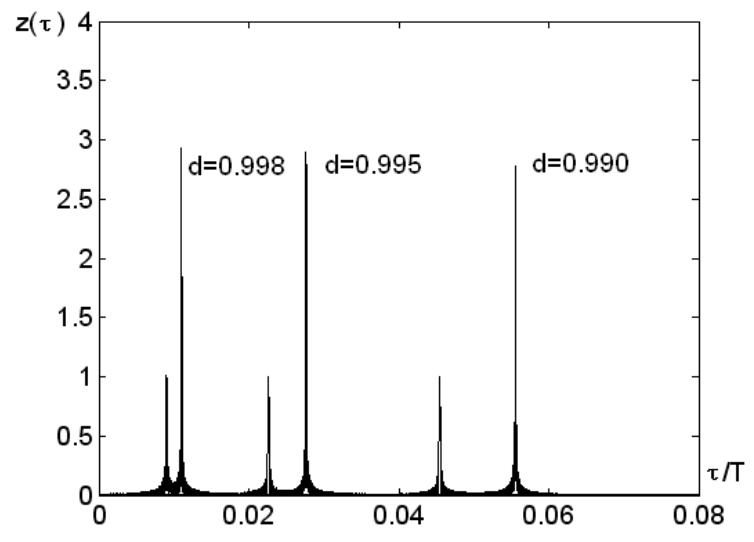

Fig. 10. HFM signal at matched filter output $\left(\tau_{0}=2.5 \mathrm{~s}, B / f_{0}=0.2\right)$.

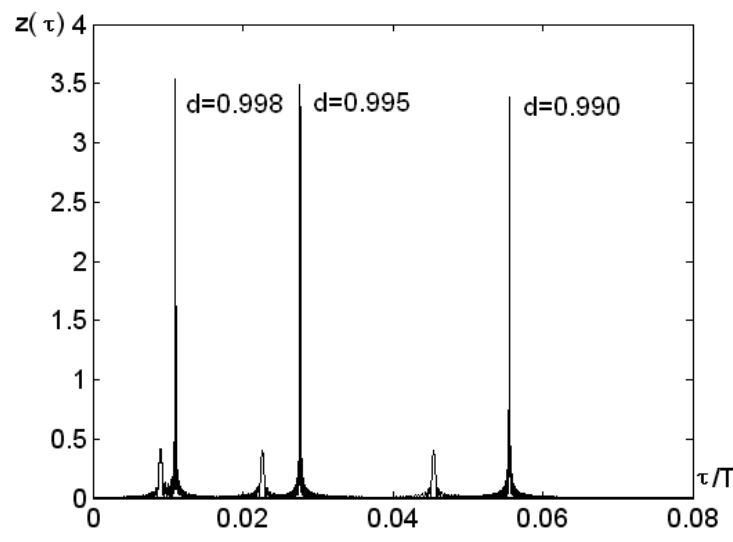

Fig.11. HFM signal at matched filter output $\left(\tau_{0}=1 \mathrm{~s}, B / f_{0}=0.2\right)$.

The delay sign $\tau$ depends on the direction of speed and the changes in the sounding signal frequency. In the examples above, the target was moving away from the sonar and frequency was rising with time. Fig. 12 shows a situation in which ascending and descending frequency signals are emitted consecutively. The first target located at distance $R=1 \mathrm{~km}$ is moving away from the sonar at $v=3.75 \mathrm{~m} / \mathrm{s}$. The second target located at distance $R=4 \mathrm{~km}$ is moving closer to the sonar at $v=7.5 \mathrm{~m} / \mathrm{s}$. As you can see, the signals are symmetrical in relation to the right target distances. This can be used to achieve a significant reduction in distance measurement errors as illustrated in Fig. 13. The targets are located at $R=1.5 \mathrm{~km}$ (the bigger one) and $R=3$ $\mathrm{km}$ (the smaller one). Dotted lines show the signals as frequencies rise and fall and the solid 
line is the result, i.e. the combination of both signals. As you can see, it occurs at target locations. False echoes occurring between the actual positions of the targets can be eliminated using signal selection which goes beyond the scope of this article.

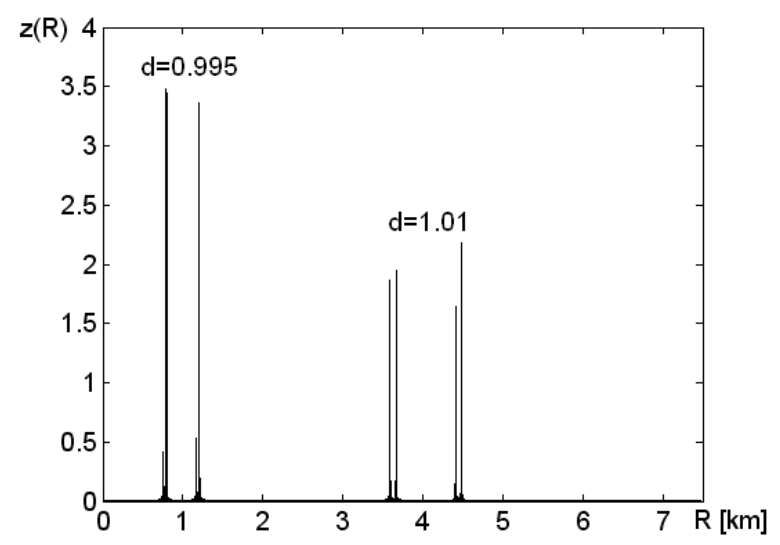

Fig. 12. HFM signal at matched filter for rising and falling frequencies $\left(T=10 \mathrm{~s}, B / f_{0}=0.2\right)$.

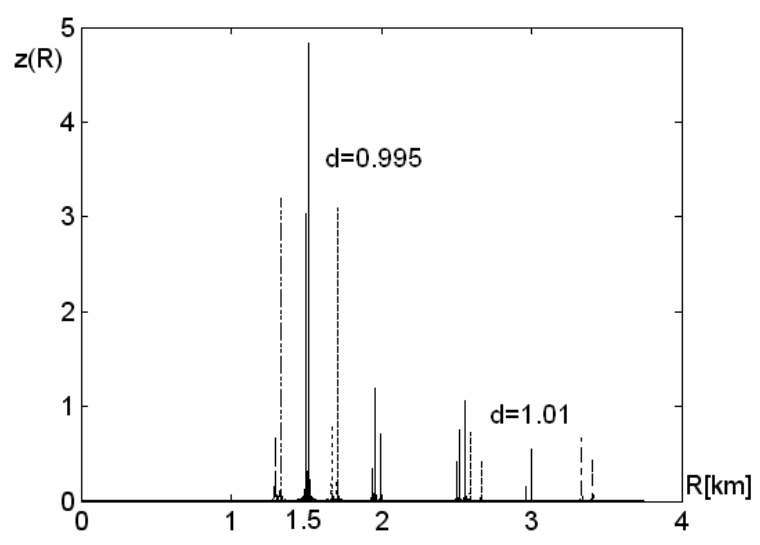

Fig. 13. Result of distance measurement error reduction $\left(T=10 \mathrm{~s}, B / f_{0}=0.2\right)$.

\section{The effects of Doppler shift on the parameters of silent sonar}

The analysis above has shown that in the case of the silent sonar distance measurement errors are primarily and most likely caused by the Doppler effect. The application and parameters of the sonar depend on whether the errors are acceptable. Errors are caused by external factors which are unknown and independent of the sonar's parameters and by those related to the sonar's parameters which we can control during the design and operation of the device. External factors include the speed and distance of targets versus the sonar. The second group of factors includes the speed of the sonar's carrier and two sonar parameters, i.e. the duration of the sounding signal $\mathrm{T}$ and its relative spectrum width $B / f_{0}$.

From the perspective of target speeds, silent sonars can be divided into those detecting stationary objects (bottom, navigation obstacles, naval mines), objects moving at low speeds (divers, underwater vehicles) and those designed to detect and follow underwater targets (submarines, torpedoes).

Stationary target sonars are affected by the Doppler effect when their medium (ship, underwater vehicle, helicopter) moves. Distance measurement errors in this case can be reduced to acceptable limits by measuring the medium's speed vector and using a rescaled sounding signal for matched filtration, which is the signal $s[d(\theta) t)]$, where $d(\theta)$ is equal to:

$$
d(\theta)=1+\frac{2}{c}|\vec{v}| \cos \theta .
$$

In the above formula $|\vec{v}|$ is the measured speed of the sonar's carrier and $\theta$ is the angle between the speed vector direction and the angle of the axis of a specific sonar beam. Modern day sonars will usually produce a number of deflected narrow beams covering a wide angular sector of simultaneous observation, [16]. Matched filtering in these sonars is performed for each beam signal separately. When compensated, the sonar's own speed does not increase the number of numerical operations performed in real time. What is necessary, however, is an additional generation of signals $s[d(\theta) t)]$, an operation that must be performed with each change of speed. Sets of the signals can be stored in computer memory.

The above method of compensating for the sonar's own speed should be applied in the majority of silent sonars, irrespective of what they are used for, because it significantly 
reduces the negative effects of the Doppler effect. Exceptions include stationary sonars which can be used as a security measure for harbours, roadstead, inland water structures, etc.

By selecting the right parameters silent sonars designed for diver and underwater vehicle observation can ensure that distance measurement errors are within acceptable limits. This can be achieved primarily thanks to slow speeds of the targets and small sonar ranges. The speed of a diver carrying a load is $v \cong 0.5 \mathrm{~m} / \mathrm{s}$, and $v \cong 1 \mathrm{~m} / \mathrm{s}$ without one. The speed of underwater vehicles is hardly ever above $v \cong 2 \mathrm{~m} / \mathrm{s}$. When used in the sonar's transmitting array, typical piezoelectric transducers can help emit signals that have the relative spectrum width of $B / f_{0}=0.2$. By substituting the value into formula (32), the distance measurement error can be written down as:

$$
\Delta R \cong 5 v T .
$$

Today's multiple beam pulse sonars used in similar applications offer ranges $R_{m}$ up to several hundred meters. In a silent sonar duration T must meet the inequality:

$$
T>2 R_{m} / c
$$

For range $R_{m}=750 \mathrm{~m}$, the minimal duration of $T$ is $1 \mathrm{~s}$. By inserting this value into formula (34) it ranges from $2.5 \mathrm{~m}$ for $v=0.5 \mathrm{~m} / \mathrm{s}$ to $10 \mathrm{~m}$ for $v=2 \mathrm{~m} / \mathrm{s}$. The same accuracy is perfectly sufficient when the target is a diver or an underwater vehicle.

To ensure the secretiveness of the silent sonar period $T$ should be as long as possible. What is needed is a compromise between an acceptable error in distance reading and the required secretiveness. Let us compare the silent sonar with the pulse sonar without matched filtering. Let us assume that both sonars operate at a frequency of $f_{0}=100 \mathrm{kHz}$, the silent sonar's transmitter emits HFM signals with a spectrum width of $B=20 \mathrm{kHz}$ and duration $T=1 \mathrm{~s}$, and the pulse sonar's transmitter emits signals whose duration is $\tau_{\mathrm{i}}=0.1 \mathrm{~ms}$. As a result, the required bandwidth of the pulse sonar's receiver is $B_{i}=1 / \tau_{1}=10 \mathrm{kHz}$.

Let us assume that the signal power at the input to the pulse sonar's receiver is $P_{i}$, and $P$ at the input to the silent sonar's receiver. Let us assume further that the required output signalto-noise ratio at the output of both receivers is identical and that the probability of detection and false alarm is identical in both cases. The pulse sonar's receiver detects the envelope which means that the output signal to noise ratio is almost equal to the input signal to noise ratio and amounts to:

$$
S N R_{i} \cong \frac{P_{i}}{N B_{i}}
$$

where $N$ is the power spectral density of sea noise.

The output signal-to-noise ratio in a silent sonar with matched filtering is equal to:

$$
S N R \cong \frac{P T}{N}
$$

The above relations show that the signal power ratio at sonar outputs is equal to:

$$
\frac{P_{i}}{P} \cong T B_{i} \text {. }
$$

For the above values the quotient is $10^{4}$ which means that the silent sonar's range is similar to that of the pulse radar but emits $10^{4}$ less power. This is a slightly overestimated figure due to the Doppler effect and its impact on the output signal.

With different powers emitted by the sonars in question, the ranges of their intercept by the enemy passive system can vary significantly. Let us assume that the passive receiver's input signal power needed for intercept is identical for signals emitted by the pulse and silent sonar. 
For spherical propagation the equation is as follows:

$$
\frac{P_{i} r_{1}^{2}}{r_{i}^{2} \exp \left(0,23 \alpha r_{i}\right)}=\frac{P r_{1}^{2}}{r^{2} \exp (0,23 \alpha r)},
$$

where $r_{i}$ means the interception range of a sounding signal emitted by the pulse sonar, $r$ - the interception range of a sounding signal emitted by the silent sonar, $r_{l}=1 \mathrm{~m}$, and $\alpha$ is the absorption coefficient of sound in water $[\mathrm{dB} / \mathrm{m}]$.

Using the relation (38), equation (39) can be written down as:

$$
T B_{i}=\frac{r_{i}^{2}}{r^{2}} \exp \left[0,23 \alpha\left(r_{i}-r\right)\right]
$$

By solving the equation numerically we can estimate the interception range of the pulse sonar in the function of silent sonar interception range. Fig. 14 shows this relation for $T B_{i}=10^{4}$ and three values of the absorption coefficient. In oceanic water at a frequency of $f_{0}=100 \mathrm{kHz}$ the value of the coefficient is $\alpha \cong 30[\mathrm{~dB} / \mathrm{km}], \alpha \cong 4.3$ in the Baltic and $\alpha \cong 1.3$ in fresh water, $[11,14]$. Because acoustic wave attenuation is very high in oceanic water, the differences between silent sonar and pulse sonar interception ranges are small. This increases in the brackish water of the Baltic Sea and even more so in inland waters.

Primarily designed for detecting submarines, long-range sonars operate at low frequencies where absorption of sound is significantly weaker. This has a positive effect on the difference between the silent sonar and pulse sonar interception ranges as illustrated in Fig. 15. The sonars in question operate at a frequency of $f_{0}=10 \mathrm{kHz}$, bandwidth $B=2 \mathrm{kHz}$ and duration $T=20 \mathrm{~s}$ $\left(R_{m}=15 \mathrm{~km}\right)$. The absorption coefficient is now $\alpha \bumpeq 0.65[\mathrm{~dB} / \mathrm{km}], \alpha \bumpeq 0.09$ in the Baltic Sea and $\alpha \cong 0.009$ in fresh water.

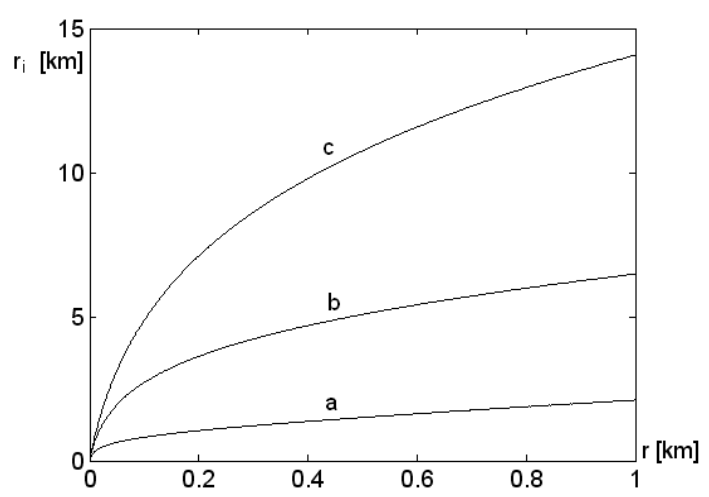

Fig. 14. Interception range of the $100 \mathrm{kHz}$ pulse sonar versus silent sonar (a- ocean, b- Baltic Sea, c- fresh water).

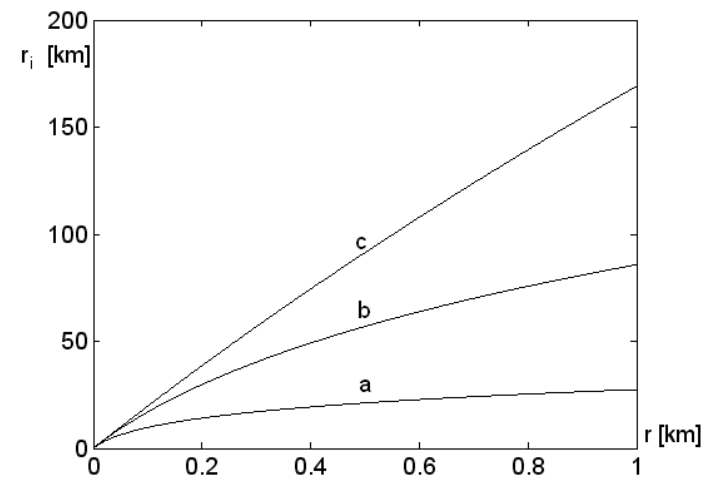

Fig. 15. Interception range of the $10 \mathrm{kHz}$ pulse sonar versus silent sonar (a- ocean, b- Baltic Sea, c- fresh water).

The tactical speed of submarines when under water ranges from $5 \mathrm{~m} / \mathrm{s}$ (older ships) to 13 $\mathrm{m} / \mathrm{s}$ (most recent ships). Combined with speeds like these, the long duration of $T$ is the source of serious distance measurement errors. Formula (34) shows that they will range from $500 \mathrm{~m}$ to $1300 \mathrm{~m}$ for the above speeds. The error can be reduced by increasing the width of the sounding signal spectrum, a method made possible by today's broadband transducer technology. The range of the silent sonar can also be reduced to ensure that it stays secretive. As an example, for $B / f_{0}=0.5$ and $T=10$, the errors can be reduced to $100 \mathrm{~m}$ and $260 \mathrm{~m}$ which is acceptable for distances of ship detection. A radical improvement in distance measurement 
accuracy can be achieved by applying algorithms using the echo signal symmetry as shown in Fig. 13.

\section{Conclusions}

The main reason why silent sonars misread the distances is the Doppler effect. These errors have acceptable values for high frequency short range sonars designed for detecting divers and underwater vehicles. The high sound absorption makes the use of such sonar particularly advantageous in waters with low salinity. The advantage of low frequency long-range silent sonars over pulse sonars is that they are more secretive. The drawback, however, is that they make serious mistakes in measuring distance, a feature which can be limited by using additional post-detection processing of echo signals. This article is limited to the analysis of the impact of the Doppler effect on the accuracy of distance measurement. This problem can be considered more broadly, for example, using the method described in article [17].

\section{Acknowledgements}

This work was supported by the Ministry of Science and Higher Education, Poland (grant ON517 624239).

\section{References}

[1] Kay, L. (1959). A comparison between pulse and frequency-modulation echo-ranging system. Journal Brit.I.R.E., 19(2), 105-113.

[2] Kay, L. (1959). An experimental comparison between pulse and frequency-modulation echo-ranging system. Journal Brit.I.R.E., 1960, 20(10), 785-796.

[3] Fuller, K.L. (1990). To see and not be seen. IEE Proceedings-F, 137(1), 1-10.

[4] Griffiths, H.D. (1990). New ideas in FM Radar. Electron. Commun. Eng. Journal, 2(5), 185-194.

[5] Skolnik, M. (1990). Radar Handbook. Second Edition. McGraw-Hill.

[6] Stove, A.G. (1992). Linear FMCW Radar Techniques. IEE Proceedings-F, 139(5), 343-350.

[7] Kramer, S.A. (1967). Doppler and acceleration tolerance of high-gain wide-band linear FM correlation SONAR. Proc. IEEE, 33, 627-636.

[8] Marszal, J., Salamon, R., Zachariasz, K., Schmidt, A. (2011). Doppler effect in CW FM sonar. Hydroacoustics, 14, 157-164.

[9] Salamon, R., Marszal, J., Schmidt, J., Rudnicki, M. (2011). Silent sonar with matched filtration. Hydroacoustics, 14, 199-208.

[10] Levanon, N., Mozeson, E. (2004). Radar signals. John Wiley\&Sons. New Jersey.

[11] Speiser, J.M. (1967). Wide-band ambiguity function. IEEE Trans. Inform. Theory, IP-13, 122-129.

[12] Lathi, B.P., Ding, Z. (2010). Modern digital and analog communication systems. Oxford University Press. New York.

[13] Hodges R. P. (2010). Underwater acoustics. John Wiley\&Sons.

[14] Kroszczyński, J.J. (1969). Pulse compression by means of linear-period modulation. Proc. IEEE 57, 1260-1266.

[15] Yang, J., Sarkar, T.K. (2006). Doppler-invariant property of hyperbolic frequency modulated waveform. Microwave and optical technology letters, 48(8), 1174-1179.

[16] Salamon, R. (2006). Sonar systems. Wyd. GTN. Gdansk. (in Polish)

[17] Kowalczyk, A., Hanus, R., Szlachta, A. (2011). Investigation of the statistical method of time delay estimation based on conditional averaging of delayed signal. Metrol. Meas. Syst., 18(2), 335-342. 